\title{
What Kind of Argument Are We Going to Have Today?
}

\author{
Eva Cogan and Simon Parsons \\ Dept of Computer and Information Science \\ Brooklyn College \\ City University of New York \\ Brooklyn, NY 11210 USA \\ \{cogan,parsons\}@sci.brooklyn.cuny.edu
}

\author{
Peter McBurney \\ Department of Computer Science \\ University of Liverpool \\ Chadwick Building \\ Peach Street, Liverpool L69 7ZF, UK \\ p.j.mcburney@csc.liv.ac.uk
}

\begin{abstract}
This paper is concerned with argumentation-based dialogues between agents. Much work in this area has been based upon an influential taxonomy of dialogue types developed by Walton and Krabbe. This paper re-examines the Walton and Krabbe framework, concentrating on the preconditions for different types of dialogue and analyzing these in a systematic way. Doing so uncovers some inconsistencies in previous interpretations of the preconditions and, in resolving these inconsistencies, identifies a number of new kinds of dialogue. We discuss some of the more interesting of these new kinds of dialogue and give protocols for them.
\end{abstract}

\section{Categories and Subject Descriptors}

I.2.11 [Artificial Intelligence]: Distributed Artificial IntelligenceCoherence and co-ordination; multiagent systems.

\section{General Terms}

Languages, theory.

\section{Keywords}

Agent communication, dialogue games, argumentation.

\section{INTRODUCTION}

Starting with Sycara [26, 27], there has been increasing interest in inter-agent dialogues that are based around the use of argumentation, that is the exchange of reasons in favor of and against the assertions of the dialogue participants. Particularly influential has been the work of Walton and Krabbe [28].

Walton and Krabbe distinguished six basic forms of dialogue: Information seeking Dialogues, where one participant seeks the answer to some question(s) from another participant, who is believed by the first to know the answer(s); Inquiry Dialogues, where the participants collaborate to answer some question or questions whose answers are not known to any one participant; Persuasion Dialogues, where one party seeks to persuade another party to adopt

Permission to make digital or hard copies of all or part of this work for personal or classroom use is granted without fee provided that copies are not made or distributed for profit or commercial advantage and that copies bear this notice and the full citation on the first page. To copy otherwise, to republish, to post on servers or to redistribute to lists, requires prior specific permission and/or a fee.

AAMAS'05, July 25-29,2005, Utrecht, Netherlands.

Copyright 2005 ACM 1-59593-094-9/05/0007 ...\$5.00. a belief or point-of-view (s)he does not currently hold; Negotiation Dialogues, where the participants bargain over the division of some scarce resource; Deliberation Dialogues, where participants collaborate to decide what course of action to take; and Eristic Dialogues, where participants quarrel verbally as a substitute for physical fighting. Furthermore, Walton and Krabbe argued that these fundamental types of dialogue could be combined, embedding, for example, a persuasion dialogue into a negotiation. Starting with Reed [24] a number of authors have taken Walton and Krabbe's framework as a starting point for discussing various kinds of dialogue.

For example, $[4,12,23]$ have discussed persuasion dialogues, [17] considered inquiries, [16, 19] looked at negotiation, and [15] examined information seeking dialogues. Others have studied more general frameworks - $[20,21]$, for example, have defined simple protocols for persuasion, information seeking and inquiry dialogues and investigated their properties. Other efforts, include the investigation, by $[6,11,25,29]$ for example, of types of dialogue that are not covered by Walton and Krabbe (who make no claims of comprehensiveness).

This paper continues the latter line of work, but goes about it in a more systematic way than, so far as we are aware, other authors have proceeded. This work is a step towards a comprehensive classification that will allow agents to select which of a broad range of dialogue types best suit their dialogical needs.

\section{BACKGROUND}

\subsection{Argumentation}

We start with the formal system of [20,21], which, for lack of space, we present very briefly, and mainly informally. A full, formal, description is in $[20,21]$ - this system also deals with preferences between arguments, which, for simplicity, we ignore here.

Each agent involved in a dialogue has a knowledge base $\Sigma$ which contains formulas of a propositional language $\mathcal{L}$. $\vdash$ stands for classical inference and $\equiv$ for logical equivalence. The main concept with which we are concerned is that of an argument. An argument is a pair $A=(S, p)$ where $p$ is a formula of $\mathcal{L}$ and $S$ a consistent subset of $\Sigma$ such that $S \vdash p$; and no proper subset of $S$ does so. $S$ is called the support of $A$, written $S=\operatorname{Support}(A)$ and $p$ is the conclusion of $A$, written $p=$ Conclusion $(A)$.

Two arguments may conflict. More precisely arguments may undercut one another, where argument $A_{1}$ undercuts $A_{2}$ iff $\exists p \in$ $\operatorname{Support}\left(A_{2}\right)$ such that $\neg p \equiv$ Conclusion $\left(A_{1}\right)$. In other words, an argument is undercut if and only if there is another argument which has as its conclusion the negation of an element of the support for the first argument. There are, of course, other ways to define a system of argumentation. This is just one approach, based on [1, 
$B_{X} p \quad p$ is the conclusion of an acceptable argument

$\mathrm{OG}_{X} p \quad p \in O B L(X)$

$I_{X} p \quad p \in I N T(X)$

$D_{X} p \quad p \in D E S(X)$

$W_{X} p \quad B_{X} p \vee B_{X} \neg p$

$A_{X, Y} p \quad\left(B_{X} p \wedge B_{Y} p\right) \vee\left(B_{X} \neg p \wedge B_{Y} \neg p\right)$

Table 1: Notation.

2], which itself is based on [7], and which our experience suggests is an adequate framework for handling agent communication.

Now, a set of arguments $\mathcal{S}$ defends an argument $A$ iff for each argument $B$ that undercuts $A$, there is an argument in $\mathcal{S}$ that undercuts $B$. From this notion we can develop the important idea of an acceptable argument. An acceptable argument $A$ is one that is not undercut, or for which there is an acceptable argument that undercuts each of the arguments that undercut $A$. An acceptable argument is one which is, in some sense, proven since all the arguments which might undermine it are themselves undermined. However, this status can be revoked following the discovery of a new argument (possibly as the result of the communication of some new information from another agent).

\subsection{Agents and dialogue}

We build a model of dialogue on top of this system of argumentation. We consider dialogues to take place between two agents called $P$ and $C$ (Pro and Con). Each agent has a private knowledge base. One part of this, the belief base, $B E L(P)$ and $B E L(C)$ respectively, contains the agent's beliefs. In addition, each agent $x \in\{P, C\}$ has a set of obligations, intentions and desires, denoted by $O B L(x), \operatorname{INT}(x)$ and $D E S(x)$ respectively, which are modeled as a multi-context system as in [19]. Such a system can take care of nested modalities and the necessary constraints between modalities, as described in [19]. Our agents are BOID agents in the sense of [5] - though we don't require obligations or desires for the work described here, we keep them in the model for continuity with our other work. We write $\mathrm{O} G_{X} p$ to denote $p \in O B L(X)$, and similarly $I_{X} p$ to denote $p \in I N T(X), D_{X} p$ to denote $p \in D E S(X)$ and $B_{X} p$ to denote that $p$ is the conclusion of an acceptable argument ${ }^{1}$. The commitment store, denoted by $C S(x)$, is a subset of the knowledge base, accessible to both agents, containing the commitments made during the dialogue. Following Hamblin [14] we take commitments to be propositions for which an agent is prepared to provide an argument.

We further define the notion of " $X$ knows whether or not $p$ ", $W_{X} p$, which denotes $B_{X} p \vee B_{X} \neg p$, and a simple notion of agreement ( $X$ and $Y$ agree about $p), A_{X, Y} p$, which denotes $\left(B_{X} p \wedge B_{Y} p\right) \vee\left(B_{X} \neg p \wedge\right.$ $\left.B_{Y} \neg p\right)$. Table 1 summarizes our notation.

\subsection{Locutions}

Next we define the locutions (moves in the dialogue game) that are available to agents. Some of the moves we use here were first introduced in [20] and modified in [22]. Each locution has a rule describing how to update the commitment stores after the move. For all moves, player $P$ addresses the $i$ th move of the dialogue to player $C$.

The first pair of moves allow propositions to be asserted.

$\operatorname{assert}(p)$ where $p$ is a propositional formula.

$$
C S_{i}(P)=C S_{i-1}(P) \cup\{p\} \text { and } C S_{i}(C)=C S_{i-1}(C)
$$

\footnotetext{
${ }^{1}$ Any proposition $p$ in $\mathcal{L}$ is the conclusion of an argument $(\{p\}, p)$.
}

Here $p$ can be any propositional formula, as well as the special character $\mathcal{U} . \mathcal{U}$ indicates that $P$ cannot give an answer.

$\operatorname{assert}(S)$ where $S$ is a set of formulas representing the support of an argument.

$$
C S_{i}(P)=C S(P)_{i-1} \cup S \text { and } C S_{i}(C)=C S_{i-1}(C)
$$

The counterpart of these moves are the acceptance moves.

$\operatorname{accept}(p) p$ is a propositional formula.

$$
C S_{i}(P)=C S_{i-1}(P) \cup\{p\} \text { and } C S_{i}(C)=C S_{i-1}(C)
$$

When $p$ is of the form $O G_{P} q$, accept $(p)$ adds $q$ to $P$ 's Obligations as well:

$$
O B L_{i}(P)=O B L_{i-1}(P) \cup\{q\} \text { and } O B L_{i}(C)=O B L_{i-1}(C)
$$

$\operatorname{accept}(S) S$ is a set of propositional formulas.

$$
C S_{i}(P)=C S_{i-1}(P) \cup S \text { and } C S_{i}(C)=C S_{i-1}(C)
$$

There are also moves which allow questions to be posed. In particular, we have the question locution, which can be used to query the other player about the truth of any proposition.

question $(p)$ where $p$ is a propositional formula.

$$
C S_{i}(P)=C S_{i-1}(P) \text { and } C S_{i}(C)=C S_{i-1}(C)
$$

There is also the challenge locution:

challenge $(p)$ where $p$ is a propositional formula.

$$
C S_{i}(P)=C S_{i-1}(P) \text { and } C S_{i}(C)=C S_{i-1}(C)
$$

A challenge is a means of making the other player explicitly state the argument supporting a proposition which he has previously asserted (and thus has added to his commitment store).

The preconditions for the locutions are determined by what has previously been called the attitude of an agent and the content of the agent's knowledge base. While a range of such attitudes are explored in [22], here we restrict ourselves to considering what [22] calls a thoughtful/skeptical agent; that is, one that is allowed to assert and accept only propositions for which it has an acceptable (in the sense defined above) argument. Such preconditions do not uniquely define which locutions an agent can use at a particular point in time. Additional constraints are provided by a protocol examples of the kind of protocol that we are interested in are given in [20].

\subsection{Dialogue protocols}

As mentioned above, [20] introduced some simple dialogue protocols. In order to contrast these with the ones we introduce, we restate the protocols from [20]. In addition, we formalize the preconditions that [20] states informally. Before giving these protocols, however, we first introduce a macro to represent a common set of locutions for the "challenge and defense" of a proposition. One agent challenges an assertion, the other provides the support for an argument for the assertion, and the first may then (where necessary) challenge any element of the justification.

$$
C D(X, Y, p)
$$

\section{1. $Y$ challenges $p$}


2. $\left\{\begin{array}{cc}X \text { asserts } S, \text { the support of } & \\ \text { an argument for } p & \text { if allowed, } \\ \text { dialogue terminates unsuccessfully } & \text { otherwise. }\end{array}\right.$

3. for each $s \in S \begin{cases}Y \text { accepts } s & \text { if allowed } \\ C D(X, Y, s) & \text { otherwise }\end{cases}$

4. $Y$ accepts $p$

We now give the protocols from [20] using $C D(X, Y, p)$. Note that all the preconditions are drawn up from the perspective of $A$, the agent that utters the first locution in any dialogue using the protocols.

Information $\operatorname{seek}(A, B, p)$. This is a dialogue in which $A$ queries $B$ about the truth of $p$ :

preconditions:

$$
\begin{aligned}
& \text { - } \neg W_{A} p \\
& \text { - } I_{A} W_{A} p \\
& \text { - } \neg B_{A} \neg W_{B} p
\end{aligned}
$$

1. A questions $(p)$

2. $\begin{cases}B \text { asserts } p & \text { if allowed, } \\ B \text { asserts } \neg p & \text { if allowed, } \\ B \text { asserts } \mathcal{U} & \text { otherwise. }\end{cases}$

3. $\begin{cases}A \text { accepts } B^{\prime} \text { s response } & \text { if allowed, } \\ C D(B, A, B \text { 'sresponse }) & \text { otherwise. }\end{cases}$

Inquiry $(A, B, p)$ is a dialogue in which $A$ and $B$ attempt to show that $p$ is true.

preconditions:

- $\neg W_{A} p$

- $I_{A} A_{A, B} p$

- $B_{A} \neg W_{B} p$

- $B_{A} I_{B} A_{A, B} p$

1. A asserts $q \rightarrow p$ for some $q$, or $\mathcal{U}$.

2. $\begin{cases}B \text { accepts } q \rightarrow p & \text { if allowed, } \\ C D(A, B, q \rightarrow p) & \text { otherwise }\end{cases}$

3. $B$ asserts $q$, or $r \rightarrow q$ for some $r$, or $\mathcal{U}$.

4. $\begin{cases}A \text { accepts } B \text { 's assertion } & \text { if allowed, } \\ C D(B, A, B \text { 's assertion }) & \text { otherwise }\end{cases}$

5. If $\mathcal{A}(C S(A) \cup C S(B))$ includes an argument for $p$ which is acceptable to both agents, then first $A$ and then $B$ accepts it and the dialogue terminates successfully.

6. Go to 3, reversing the roles of $A$ and $B$ and substituting $r$ for $q$ and some $t$ for $r$.
Persuade $(A, B, p)$ is a dialogue in which $A$ attempts to persuade $B$ that $p$ is the case.

preconditions:

- $B_{A} p$

- $B_{A} \neg B_{B} p$

- $I_{A} B_{B} p$

1. A asserts $p$

2. $\begin{cases}B \text { accepts } p & \text { if allowed, } \\ B \text { asserts } \neg p & \text { if allowed, } \\ C D(B, A, p) & \text { otherwise. }\end{cases}$

3. If $B$ asserts $\neg p$, then go to 2 with the roles reversed and $\neg p$ instead of $p$.

We now turn to the main contribution of this paper, which is to examine the preconditions of different kinds of dialogue.

\section{PREREQUISITES FOR DIALOGUE}

We can summarize Walton and Krabbe's [28, pages 65-85] descriptions of the three dialogue types that deal with beliefs (which will be our focus here) as:

Information seeking Dialogues: One participant has some information, or is in a position to know it, and the other both does not have the information and needs it. Both participants share the goal of spreading knowledge.

Inquiry Dialogues: The participants collaborate to answer some question or questions whose answers are not known to any one participant. Both parties are initially ignorant about the answer, but are committed to resolving the question.

Persuasion Dialogues: One party seeks to persuade another party to adopt a belief or point-of-view (s)he does not currently hold. These dialogues begin with one party supporting a particular statement which the other party to the dialogue does not hold, and the first seeks to resolve the conflict by convincing the second to adopt the proposition. The second party shares the objective of resolving the conflict, but may try to do this by convincing the other to change his or her mind.

One way to interpret Walton and Krabbe's descriptions is in terms of the conditions that hold at the beginning and end of a specific kind of dialogue. In the literature this has typically been done in the sense of defining the initial conditions that any dialogue protocol must cope with, and the final conditions it must bring about to be successful (for example as in [3]). Thus, since an inquiry dialogue starts with no one participant knowing whether or not the proposition in question is true, and will end successfully with a proof of the proposition or its negation, the minimum requirement for an inquiry dialogue is that it must be able to construct a proof where the various components are distributed among the participants, exactly as in the Inquiry protocol given above.

Another approach, and the one we pursue here, is to consider the preconditions as a guide to the participants as to what kind of dialogue it is appropriate to engage in. Thus if Shimon is ignorant about the truth of $p$ and needs to know it, then if Piotr knows whether $p$, it makes sense for Shimon to engage Piotr in an information seeking dialogue, while if Piotr does not know $p$, then it makes sense for Shimon to engage him in an inquiry into $p$. From this 


\begin{tabular}{l|lll} 
& $B_{B} p$ & $B_{B} \neg p$ & $\neg W_{B} p$ \\
\hline$B_{A} p$ & & Persuasion & Information seeking \\
$B_{A} \neg p$ & Persuasion & & Information seeking \\
$\neg W_{A} p$ & Information seeking & Information seeking & Inquiry
\end{tabular}

Table 2: Preconditions from Walton and Krabbe [13, 28].

\begin{tabular}{|c|c|c|c|c|}
\hline & & $B_{A} B_{B} \neg p \quad B_{A} I_{B} A_{A, B} p$ & $B_{A} I_{B} A_{A, B} p$ & $B_{A} W_{B} p$ \\
\hline $\begin{array}{r}B_{A} p \\
\neg W_{A} p\end{array}$ & $\begin{array}{l}I_{A} A_{A, B} p \\
I_{A} A_{A, B} p\end{array}$ & Prsuasion & Inquiry & Information seeking \\
\hline
\end{tabular}

Table 3: Modified preconditions from Walton and Krabbe.

perspective, we can think of Table 2 (which is taken from [13] and modified to mesh with our notation) as specifying which dialogue is appropriate under which conditions. As for all the tables we will come across in this paper, the preconditions are laid out along both axes. In the cells are the appropriate dialogues, and if the relevant dialogue is successful, the intention(s) of the participants will be fulfilled. A space indicates that there is no dialogue that covers the eventuality, in this case when both agents agree on the truth of a proposition.

However, neat though this characterization is, and apparently exactly what Walton and Krabbe intended, it is no use to Shimon in his efforts to determine what kind of dialogue is appropriate in determining the truth of $p$. Why not? Because he will not, in general know the truth of $B_{\text {Piotr }} p$. Instead, he has some belief about what Piotr believes - that is, he can determine if $B_{\text {Shimon }} B_{\text {Piotr }} p-$ and must use this to make his decision about the most appropriate dialogue.

Furthermore, the goal (or, as we model it here, the intention) of the participants comes into play. It is not just their mutual ignorance about $p$ that suggests Shimon should engage Piotr in an inquiry, but the fact that Shimon intends to know whether $p$ is true or not, and believes that Piotr does the same. These considerations suggest that Table 3 rather than Table 2 is what Shimon should use to determine what kind of dialogue is most appropriate. This takes the goals of the dialogue, as stated in [28] and restates them as preconditions.

Note that Table 3 deals only with the conditions from A's perspective (in other words in terms of $A$ 's beliefs, and so, like the remainder of the tables in this paper presents the perspective of the initiator of the dialogue), and exploits the symmetry in $p$ and $\neg p$. Were we to distinguish $p$ and $\neg p$ in $A$ 's beliefs we would get an expanded version which was symmetrical in $\neg p$, and we could further add a similar set of results for $B$ to get a table that included all the entries in Table 2. We leave these additional entries out here and for the remainder of the paper because they are redundant.

The table does more than tell Shimon what dialogues are appropriate in different situations; it identifies some suggestive gaps. For example, under Walton and Krabbe's definition, it isn't possible for $A$ to engage $B$ in a persuasion unless $B$ wants to resolve the inconsistency. If $B$ doesn't care, then the dialogue cannot be a persuasion. We argue that this is unnecessarily restrictive. We have all been party to persuasions where we didn't want to resolve the issue but were forced into the dialogue by some convention (reluctant encounters with authority for example, or not wishing to give too much offence to doorstopping evangelists) and from the point of view of formalization, actively requiring both participants to want to resolve the situation isn't necessary. Provided that $B$ is at least cooperative, in the sense of not actively trying to derail or prolong the dialogue ${ }^{2}$, then $A$ may rationally initiate a persuasion.

Examining the protocols given above, reveals that it is possible to relax the preconditions for persuasion and information seeking. In particular,

$$
I_{A} A_{A, B} p \wedge B_{A} I_{B} A_{A, B} p
$$

(which is a requirement in [20]) is not required for $A$ to initiate a persuasion. Under the interpretation we favor, a sufficient condition for $A$ to start a persuasion is that $I_{A} B_{B} p, A$ wants $B$ to believe $p$. Indeed, the protocol for persuasion given above also works when $B_{A} \neg W_{B} p$, that is whether or not $B$ believes anything about $p$.

For an information seeking dialogue, we suggest that the preconditions should allow $A$ to start a dialogue whether or not $A$ believes he knows $B$ 's position on $p$, as long as $\neg W_{A} p$ ( $A$ doesn't currently have a position on $p$ ). Once again, the protocol works under these conditions, and it seems a sensible relaxation. Some information seeking dialogues make sense under such condition. An example of such a dialogue is one in which I stop random people in the street to ask directions when hopelessly lost - I have no idea whether they know the place to which I am headed, but I might still want to ask them.

With these new preconditions, Table 3 expands to become Table 4 . Note that the preconditions given are not those as stated in [20], but are consistent with the dialogues given there.

\section{NEW DIALOGUES AND PROTOCOLS}

Despite this relaxation of the initial conditions, there are several situations in which it seems natural to engage in dialogues, but to which the basic Walton and Krabbe dialogue types do not apply. In this section we identify some of these, and give protocols that capture them, extending the set of protocols given in [20]. Note that we are not claiming that we are identifying all possible dialogues here (one could, of course, continue modifying preconditions more or less forever), rather that by carefully considering the preconditions we can identify some useful kinds of dialogue that are apparently not included in the Walton and Krabbe classification (dialogues, therefore, that it might not be possible to engage in under a strict implementation of the Walton and Krabbe typology).

To start with, we note that as things stand, an agent is allowed to engage in information seeking and inquiry dialogues only if it is ignorant (to use Walton and Krabbe's [28, page 66] terminology) about the subject of the dialogue. The only kind of dialogue about $p$ in which one can engage when one knows $p$, according to Walton and Krabbe, is a persuasion. However, there are cases in which it

\footnotetext{
${ }^{2}[8,9,10]$ give examples of cases where one would not want to do this, for example when engaged in a dialogue with law enforcement officers who wish to persuade one to confess to a crime.
} 


\begin{tabular}{rl|ccccc} 
& & $B_{A} B_{B} p$ & $B_{A} B_{B} \neg p$ & $B_{A} \neg W_{B} p$ & $B_{A} \neg W_{B} p$ & $B_{A} W_{B} p$ \\
& & & $B_{A} I_{B} A_{A, B} p$ & & \\
\hline$B_{A} p$ & $I_{A} B_{B} p$ & & Persuasion & Persuasion & Persuasion & \\
$\neg W_{A} p$ & $I_{A} A_{A, B} p$ & Information seeking & Information seeking & Inquiry & & Information seeking
\end{tabular}

Table 4: Modified preconditions from Parsons, Wooldridge and Amgoud [22].

is natural to have other kinds of dialogue about some $p$ that one believes to be true.

Consider that Shimon believes some proposition $p$ to be true ( $p$ might be the proposition that "According to Walton and Krabbe, both participants in a persuasion dialogue have to start the dialogue with opinions about the subject of the dialogue"), but wants to check whether he is correct by asking Piotr if he thinks this is the case. This would be an information seeking dialogue if Shimon didn't already have an opinion about $p$. Since the initial conditions differ from an information seeking dialogue, we require a new dialogue type and a new protocol. We call this kind of dialogue a verification dialogue since Shimon is seeking to check that he is correct.

\subsection{Verification dialogue}

In a verification dialogue, agent $A$ seeks the answer to some question from agent $B$, and the proposition with which the dialogue is concerned is $p$. Unlike the case for information seeking, we no longer require that $A$ doesn't know $p\left(\neg W_{A} p\right)$. We require only that $A$ wants to see if $B$ thinks $p$ is true $\left(I_{A} B_{A} B_{B} p\right)$, and we don't have any condition on what $B$ believes or on what $A$ believes that $B$ believes (we are all familiar with dialogues in which we say, for instance, "do you want that last piece of cake?", thinking the answer will be "yes", but hoping it will be "no" and these seem to be verification dialogues just as much as the previous example). One possible protocol for conducting a verification dialogue about $p$ is the following. Note that all the protocols given in this paper, like those in [20], are the minimal protocol we can imagine for the task at hand.

$\operatorname{Verify}(A, B, p)$

preconditions:

$$
\text { - } I_{A} B_{A} B_{B} p
$$

1. A questions $p$

2. $\begin{cases}B \text { asserts } p & \text { if allowed, } \\ \text { dialogue terminates unsuccessfully } & \text { otherwise. }\end{cases}$

If $B$ asserts $p$, the dialog was successful.

If not, the dialogue fails. If $A$ wants to continue the discussion about $p, A$ must initiate another dialogue. For example, $A$ might then proceed to persuade $B$. Since a verification dialogue is narrowly focused on the question of whether $B$ believes $p$ or not, it is even simpler than an information seeking dialogue (which requires that $A$ be sure to check the grounds of $B$ 's argument for $p$ in order to know whether it can accept $p$ ). Thus a verification dialogue won't help $A$ if it wants to know the reason that $B$ believes $p$.

Knowing the reason may be irrelevant - as when Shimon just wants to check his facts about Walton and Krabbe. However, knowing the reason may be important. Shimon may have an argument for $p$ (where $p$ is "It is important to attend AAMAS this year") based on the fact that his friends will be there, but want to come up with a stronger one if possible (to convince the chairman of his department to pay for the trip, say). As a result Shimon may want to find out from Evelyn what she thinks the reason for the importance of AAMAS is, in case it is a better argument. Similarly, Shimon may be about to engage Piotr in a persuasion about $p$ ("Shimon should be the first author on the paper we are writing"), and might think his chances of winning the argument are improved if he obtains Piotr's reasons for his (Piotr's) position first. (He (Shimon) can then construct an argument that is less likely to be undercut.) In either case we need a form of dialogue which focuses on the argument for the subject rather than the subject itself. We call this kind of dialogue a query, and describe it in detail next.

Another common example, as suggested in [25], which distinguishes between verify and query is that of the teacher who asks the student a question to which the teacher already knows the answer. The teacher is looking to verify that the student knows the answer as well. If the teacher wants the student to defend his position, it becomes a query.

\subsection{Query dialogues}

The query dialogue arises in a situation where $A$ will always challenge after $B$ asserts its answer about $p$ because $A$ isn't interested only in whether or not $B$ believes $p$, but rather wants $B$ 's argument for $p$. This marks a shift from the underlying assumptions used in introducing the protocols in [20] — in that work agents always accepted whenever they were allowed to. For a query, agents always challenge. A simple protocol for a query dialogue is as follows:

$\operatorname{Query}(A, B, p)$

preconditions:

- $I_{A} W_{A} p$

- $\neg B_{A} \neg W_{B} p$

1. A questions $p$

2. $\begin{cases}B \text { asserts } p & \text { if allowed, } \\ \text { dialogue terminates unsuccessfully } & \text { otherwise. }\end{cases}$

3. $\mathrm{CD}(A, B, p)$

A dialogue under the Query protocol ${ }^{3}$ succeeds when $B$ offers an argument for $p$ that is acceptable to $A$. Note again that we don't require $A$ to be ignorant about $p$ before undertaking the dialogue.

We consider that the dialogue has failed if $A$ doesn't find $B$ 's argument acceptable since it has failed in its objective of discovering an argument ( $A$ 's perspective is the only one that counts here because $A$ initiated the dialogue). However, this does not mean that the dialogue need have been a waste of time for $A$. At the very least

\footnotetext{
${ }^{3}$ We will follow the convention of referring to a dialogue under a specific protocol by the name of the protocol, so that a Query dialogue is one under the Query protocol, and is distinct from a "query dialogue", which is any dialogue in the general class in which one agent is interested in the argument another has for a proposition.
} 
$A$ may have obtained some new information (some of $B$ 's grounds for $p$ ) that $A$ can use to construct a different new argument for $p$. Furthermore, if $A$ started the query to discover $B$ 's argument prior to a persuasion about $p$, then a failure might be more helpful to $A$ than a success.

That completes our discussion of Query dialogues, but there is another kind of dialogue that stands in the same relation to those generated by the Query protocol as inquiry does to information seeking. Under the conditions proposed by Walton and Krabbe, an inquiry can only take place when both agents don't know whether or not $p$ is true, and both intend to resolve the matter. There is another kind of query, a mutual query, in which $A$ and $B$ work together to establish a mutually acceptable argument for $p$, but from a position that either or both of them already has an opinion about the truth of $p$. Such a dialogue has some elements of persuasion and inquiry as defined by Walton and Krabbe, but we believe it to be subtly different enough to be a separate class of dialogue.

An example here is when Shimon and Evelyn get together to discuss their ideas for a paper on new kinds of dialogue. Evelyn believes that they have a new classification of dialogue types, and wants to check that Shimon agrees. Now, because this isn't something that Shimon has necessarily thought about prior to the meeting, Evelyn can't just question and launch into a query dialogue. In addition, Evelyn can't use an inquiry, since that requires her to not believe she has a classification before the dialogue commences. Furthermore, because what is important is not having Evelyn convince Shimon to agree, but seeing whether they can jointly build a case, it isn't a persuasion. Instead, what is required is a dialogue in which the two of them jointly construct the case for writing the paper, arguing out the truth of each step along the way, but one that allows Evelyn to have a position on the subject of the dialogue before the dialogue commences.

To cover this case we introduce a Query 2 dialogue which does exactly this. One possible protocol for it is:

Query2 $(A, B, p)$

preconditions:

$$
\begin{aligned}
& \text { - } I_{A} W_{A} p \\
& \text { - } B_{A} \neg W_{B} p
\end{aligned}
$$

1. $A$ asserts $q \rightarrow p$ for some $q$, or $\mathcal{U}$.

2. $\begin{cases}B \text { accepts } q \rightarrow p & \text { if allowed, } \\ C D(A, B, q \rightarrow p) & \text { otherwise }\end{cases}$

3. $B$ asserts $q$, or $r \rightarrow q$ for some $r$, or $\mathcal{U}$.

4. $\begin{cases}A \text { accepts } B \text { 's assertion } & \text { if allowed, } \\ C D(B, A, B \text { 's assertion }) & \text { otherwise }\end{cases}$

5. If $\mathcal{A}(C S(A) \cup C S(B))$ includes an argument for $p$ which is acceptable to both agents, then first $A$ and then $B$ accepts it and the dialogue terminates successfully.

6. Go to 3, reversing the roles of $A$ and $B$ and substituting $r$ for $q$ and some $t$ for $r$.

This completes our discussion of Query 2 , but there is yet another kind of query dialogue that we can imagine.

Going back to the case of Shimon and Evelyn's discussion about writing a paper which motivated the Query2 protocol, we recall that it started from the position that Evelyn wanted to discuss whether they had a new classification of dialogue types. We can easily imagine a situation in which Evelyn hopes that Shimon and Evelyn together might produce an acceptable argument for $p$ (in other words an argument that proves $p$ is true), rather than aiming to know the truth of $p$.

Thus Evelyn may initiate this dialogue irrespective of either participant's current position on $p$. In fact, it even makes sense to initiate this kind of dialogue when either or both participants believe the proposition to be false. Although Shimon might initially believe that there isn't a paper to be written, the discussion might end up constructing an argument for the proposition that there is one.

This seems to us to be a new kind of query dialogue, one we will call Query3, and a protocol for such a dialogue is:

Query3 $(A, B, p)$

preconditions:

$$
\text { - } I_{A} B_{A} p
$$

1. $A$ asserts $q \rightarrow p$ for some $q$, or $\mathcal{U}$.

2. $\begin{cases}B \text { accepts } q \rightarrow p & \text { if allowed, } \\ C D(A, B, q \rightarrow p) & \text { otherwise }\end{cases}$

3. B asserts $q$, or $r \rightarrow q$ for some $r$, or $\mathcal{U}$.

4. $\begin{cases}A \text { accepts } B \text { 's assertion } & \text { if allowed, } \\ C D(B, A, B \text { 's assertion }) & \text { otherwise }\end{cases}$

5. If $\mathcal{A}(C S(A) \cup C S(B))$ includes an argument for $p$ which is acceptable to both agents, then first $A$ and then $B$ accepts it and the dialogue terminates successfully.

6. Go to 3 , reversing the roles of $A$ and $B$ and substituting $r$ for $q$ and some $t$ for $r$.

An interesting kind of dialogue that is close to Query 3 is one in which a criminal lawyer and a defendant jointly seek arguments to prove that the defendant is innocent, whether or not they individually believe this to be the case. The lawyer's job in such a case is not to determine whether or not his client committed the crime but to produce a good case for the defense. The lawyer doesn't want to know all the facts that defendant knows, just those that build his case.

This completes our discussion of new dialogue types.

\subsection{A new classification}

With these new kinds of dialogue, we can fill in the gaps in Table 4. In fact, we do more than that. With these new kinds of dialogue we are covering interactions which (in terms of their preconditions) were obscured in the previous tables, identifying new goals that $A$ might have for engaging in a dialogue. The result is Table 5.

Furthermore, Table 5 also includes the result of some subtle changes to inquiry and persuasion dialogues as well. In persuasion dialogues, the weakening is to change the condition on $A$ 's beliefs about $B$ 's beliefs about $p$ so that $A$ can engage $B$ in a persuasion without even knowing that $B$ doesn't agree about $p$ (on top of the previous relaxation that $A$ no longer had to know that $B$ disagrees). Now the key thing is that $A$ believes $p$ and wants $B$ to believe it too - that, to us, seems the essence of persuasion. The change allows persuasion to encompass situations where the dialogue is "evangelical" - where $A$ wants to get other agents to agree with it because 


\begin{tabular}{cl|ccccc} 
& & $B_{A} B_{B} p$ & $B_{A} B_{B} \neg p$ & $B_{A} \neg W_{B} p$ & $B_{A} \neg W_{B} p$ & $B_{A} W_{B} p \wedge \neg B_{A} B_{B} p \wedge \neg B_{A} B_{B} \neg p$ \\
& & & $B_{A} I_{B} A_{A, B} p$ & \\
\hline$B_{A} p$ & $I_{A} B_{B} p$ & & Persuasion & Persuasion & Persuasion & Persuasion \\
$\neg W_{A} p$ & $I_{A} W_{A} p$ & Info. seeking & Info. seeking & Inquiry & Inquiry & Info. seeking \\
& $I_{A} W_{A} p$ & Query & Query & Query2 & Query2 & Query \\
& $I_{A} B_{A} p$ & Query3 & Query3 & Query3 & Query3 & Query3 \\
& $I_{A} B_{A} B_{B} p$ & & & & & Verify
\end{tabular}

Table 5: An intermediate set of preconditions

\begin{tabular}{rl|cccc} 
& & $B_{A} B_{B} p$ & $B_{A} B_{B} \neg p$ & $B_{A} \neg W_{B} p$ & $B_{A} W_{B} p \wedge \neg B_{A} B_{B} p \wedge \neg B_{A} B_{B} \neg p$ \\
\hline$B_{A} p$ & $I_{A} B_{B} p$ & & Persuasion & Persuasion & Persuasion \\
$\neg W_{A} p$ & $I_{A} W_{A} p$ & Information seeking & Information seeking & Inquiry & Information seeking \\
& $I_{A} W_{A} p$ & Query & Query & Query2 & Query \\
& $I_{A} B_{A} p$ & Query3 & Query3 & Query3 & Query3 \\
& $I_{A} B_{A} B_{B} p$ & & & & Verify
\end{tabular}

Table 6: Our preconditions

it feels so strongly that $p$ is true and wants to broadcast the fact as well as the situations that $[20,28]$ consider persuasions. Once again, the existing persuasion protocol from [20] will handle this weakening without alteration.

In inquiry dialogues, it does not seem necessary for $B$ to have the goal of establishing the truth of $p$. So long as one participant in an inquiry sets it off, all that is required of the other participant is that they respond truthfully and cooperatively when it is their turn, filling in missing pieces of the proof to the best of their ability. As a result, we drop the requirement $I_{B} A_{A, B} p$. The protocol for inquiry given above will work under this alteration to the preconditions since it makes no assumptions about $B$ 's goals.

Finally, from the perspective of $A$ trying to decide what dialogues they can engage in under specific conditions, this considerably eases $A$ 's job since it no longer has to figure out what $B$ 's intentions are. The third and fourth columns of Table 5 thus collapse, and we are left with Table 6 .

\section{DISCUSSION}

Having arrived at this new set of dialogues, and the accompanying elaboration of the preconditions (and goals encoded as preconditions), it is natural to ask "so what is this good for?" We believe that there are several answers.

To begin with, it is useful to have identified that there are these additional kinds of dialogues, which seem distinct from those proposed by Walton and Krabbe and commonly discussed in the literature. While the philosophical distinctions between these new types and the familiar information seeking, inquiry, and persuasion, are perhaps minor, the practical importance is more major. These new dialogues are themselves useful - we started on this line of work because we identified the need for the Verify dialogue in the context of work on delegation - and if we are going to build agents that engage in the dialogues we need to identify protocols by which they can so engage. Thus identifying the protocols, rather than the types of dialogue is important in the context of our wider research goals.

The desire to build agents that can engage in dialogue also explains why we have bothered to tease out the preconditions in such detail. As we have stressed throughout the paper, identifying which preconditions go with which dialogue (and hence with which protocol) is important so that an agent can choose which protocol it should make use of depending on what it knows about the agent with which it proposes to converse. Thus we see the preconditions as a necessary step towards operationalizing dialogue, and the statement of the preconditions in terms of mental notions (which Walton and Krabbe were largely careful to skirt around) is a necessary step in doing this.

\section{SUMMARY}

This paper has considered dialogues about beliefs - that is dialogues akin to the ones that Walton and Krabbe [28] called information seeking, inquiry and persuasion - and, in particular, has systematically considered the preconditions for such dialogues. Doing so has exposed a need for a number of new kinds of dialogue (Verify, Query, Query2 and Query3), and we have given protocols for these. Of course there is no more reason to think that this set of dialogues is complete, any more than there was any reason to suspect that the set originally identified by Walton and Krabbe was complete - the dialogues we have listed here, and the preconditions for them, just represent our current understanding.

Having identified these new forms of dialogue, we need to examine their properties, just as [22] did for persuasion, information seeking and inquiry dialogues, and this is something we will carry out in the near future. We also plan to continue our analysis on dialogues about actions, that is to expand into the territory of the kinds of dialogue that Walton and Krabbe called deliberation and negotiation.

As the different forms of dialogue multiply, it seems increasingly likely that we will not directly program agents with a range of different protocols of the kind described in this paper. Instead, we will program agents with the kinds of atomic protocols discussed in [18] - sub-protocols, like $C D$, from which more complex protocols can be constructed. These atomic protocols will then be used to construct the kinds of protocol described here, enabling agents to verify, query, persuade, inquire and information seek. However, in order to do this, we need to develop rules for composing atomic protocols to build up a range of complex interactions, and how to do this is a topic of our ongoing work.

\section{Acknowledgements}

This work was made possible by funding from NSF \#REC-0219347, NSF \#IIS 0329037, EU FP6-IST 002307 (ASPIC) and PSC- 
CUNY Award \#66171-00 35. The authors are grateful to the anonymous referees for their constructive comments on the paper and to Rohit Parikh for a comment that led to one of our examples.

\section{REFERENCES}

[1] L. Amgoud. Contribution a l'integration des préferences dans le raisonnement argumentatif. $\mathrm{PhD}$ thesis, Université Paul Sabatier, Toulouse, July 1999.

[2] L. Amgoud and C. Cayrol. On the acceptability of arguments in preference-based argumentation framework. In Proceedings of the 14th Conference on Uncertainty in Artificial Intelligence, pages 1-7, 1998.

[3] L. Amgoud, N. Maudet, and S. Parsons. Modelling dialogues using argumentation. In E. Durfee, editor, Proceedings of the Fourth International Conference on Multi-Agent Systems, pages 31-38, Boston, MA, USA, 2000. IEEE Press.

[4] T. J. M. Bench-Capon, F. P. Coenen, and P. Orton. Argument-based explanation of the British Nationality Act as a logic program. Computers, Law and AI, 2(1):53-66, 1993.

[5] Jan Broersen, Mehdi Dastani, Joris Hulstijn, Zisheng Huang, and Leendert der van Torre. The BOID architecture: conflicts between beliefs, obligations, intentions and desires. In Jörg P. Müller, Elisabeth Andre, Sandip Sen, and Claude Frasson, editors, Proceedings of the Fifth International Conference on Autonomous Agents, pages 9-16, Montreal, Canada, 2001. ACM Press.

[6] F. Dignum, B. Dunin-Kęplicz, and R. Verbrugge. Agent theory for team formation by dialogue. In C. Castelfranchi and Y. Lespérance, editors, Seventh Workshop on Agent Theories, Architectures, and Languages, pages 141-156, Boston, USA, 2000.

[7] P. M. Dung. On the acceptability of arguments and its fundamental role in nonmonotonic reasoning, logic programming and $n$-person games. Artificial Intelligence, 77:321-357, 1995.

[8] P. E. Dunne. Prevarication in dispute protocols. In G. Sartor, editor, Proceedings of the Ninth International Conference on AI and Law (ICAIL-03), pages 12-21, New York, NY, USA, 2003. ACM Press.

[9] D. M. Gabbay and J. Woods. More on non-cooperation in Dialogue Logic. Logic Journal of the IGPL, 9(2):321-339, 2001.

[10] D. M. Gabbay and J. Woods. Non-cooperation in Dialogue Logic. Synthese, 127(1-2):161-186, 2001.

[11] R. Girle. Commands in Dialogue Logic. In D. M. Gabbay and H. J. Ohlbach, editors, Practical Reasoning: Proceedings of the First International Conference on Formal and Applied Practical Reasoning (FAPR 1996), Bonn, Germany, Lecture Notes in Artificial Intelligence 1085, pages 246-260, Berlin, Germany, 1996. Springer.

[12] T. F. Gordon. The Pleadings Game: An exercise in computational dialectics. Artificial Intelligence and Law, 2:239-292, 1994.

[13] K. Greenwood, T. Bench-Capon, and P. McBurney. Structuring dialogue between the People and their representatives. In R. Traunmüller, editor, Electronic Government: Proceedings of the Second International Conference (EGOV03), Prague, Czech Republic, Lecture Notes in Computer Science 2739, pages 55-62, Berlin, Germany, 2003. Springer.

[14] C. L. Hamblin. Fallacies. Methuen and Co Ltd, London, UK, 1970.
[15] J. Hulstijn. Dialogue Models for Inquiry and Transaction. $\mathrm{PhD}$ thesis, Universiteit Twente, Enschede, The Netherlands, 2000.

[16] S. Kraus, K. Sycara, and A. Evenchik. Reaching agreements through argumentation: a logical model and implementation. Artificial Intelligence, 104(1-2):1-69, 1998.

[17] P. McBurney and S. Parsons. Representing epistemic uncertainty by means of dialectical argumentation. Annals of Mathematics and Artificial Intelligence, 32(1-4):125-169, 2001.

[18] S. Parsons, P. McBurney, and M. Wooldridge. The mechanics of some formal inter-agent dialogue. In F. Dignum, editor, Advances in Agent Communication. Springer-Verlag, Berlin, Germany, 2003.

[19] S. Parsons, C. Sierra, and N. R. Jennings. Agents that reason and negotiate by arguing. Journal of Logic and Computation, 8(3):261-292, 1998.

[20] S. Parsons, M. Wooldridge, and L. Amgoud. An analysis of formal inter-agent dialogues. In 1st International Conference on Autonomous Agents and Multi-Agent Systems. ACM Press, 2002.

[21] S. Parsons, M. Wooldridge, and L. Amgoud. On the outcomes of formal inter-agent dialogues. In $2 n d$ International Conference on Autonomous Agents and Multi-Agent Systems. ACM Press, 2003.

[22] S. Parsons, M. Wooldridge, and L. Amgoud. Properties and complexity of formal inter-agent dialogues. Journal of Logic and Computation, 13(3):347-376, 2003.

[23] H. Prakken. Relating protocols for dynamic dispute with logics for defeasible argumentation. Synthese, 127:187-219, 2001.

[24] C. Reed. Dialogue frames in agent communications. In Y. Demazeau, editor, Proceedings of the Third International Conference on Multi-Agent Systems, pages 246-253. IEEE Press, 1998.

[25] E. Sklar and S. Parsons. Towards the application of argumentation-based dialogues for education. In C. Sierra and E. Sonenberg, editors, Proceedings of the 3rd International Conference on Autonomous Agents and Multi-Agent Systems. IEEE Press, 2004.

[26] K. Sycara. Argumentation: Planning other agents' plans. In Proceedings of the Eleventh Joint Conference on Artificial Intelligence, pages 517-523, 1989.

[27] K. Sycara. Persuasive argumentation in negotiation. Theory and Decision, 28:203-242, 1990.

[28] D. N. Walton and E. C. W. Krabbe. Commitment in Dialogue: Basic Concepts of Interpersonal Reasoning. State University of New York Press, Albany, NY, USA, 1995.

[29] T. Yuan, D. Moore, and A. Grierson. Educational human-computer debate: A computational dialectics approach. In G. Carenini, F. Grasso, and C. Reed, editors, Proceedings of the Workshop on Computational Models of Natural Argument, 2002. 